\title{
Análise espacial das condições de enfrentamento à COVID-19: uma proposta de Índice da Infraestrutura da Saúde do Brasil
}

\author{
Spatial analysis of Brazil's COVID-19 response capacity: \\ a proposal for a Healthcare Infrastructure Index
}

Évilly Carine Dias Bezerra (https://orcid.org/0000-0002-9876-6260) ${ }^{1}$

Priscila Soares dos Santos (https://orcid.org/0000-0002-7313-3651) ${ }^{1}$

Fernanda Cigainski Lisbinski (https://orcid.org/0000-0001-9131-5996) ${ }^{1}$

Lázaro Cezar Dias (https://orcid.org/0000-0003-1051-6054) ${ }^{1}$
${ }^{1}$ Programa de PósGraduação em Economia e Desenvolvimento, Universidade Federal de Santa Maria. Av. Roraima 1000, Cidade Universitária. 97105-900 Santa Maria RS Brasil. evillycarine@hotmail.com

\begin{abstract}
One of the concerns linked to the COVID-19 pandemic is the capacity of health systems to respond to the demand for care for people with the disease. The objective of this study was to create a COVID-19 response Healthcare Infrastructure Index (HII), calculate the index for each state in Brazil, and determine its spatial distribution within and across regions. The HII was constructed using principal component factor analysis. The adequacy of the statistical model was tested using the Kaiser-Meyer-Olkin test and Bartlett's test of sphericity. The spatial distribution of the HII was analyzed using exploratory spatial data analysis. The data were obtained from DATASUS, the Federal Nursing Council, Ministry of Health, Government Procurement Portal, and the Transparency Portal. The nine states in the country's North and Northeast regions showed the lowest indices, while the five states from the Southeast and South regions showed the highest indices. Low-low clusters were observed in Amazonas and Pará and high-high clusters were found in Minas Gerais, Rio de Janeiro, São Paulo, and Paraná.
\end{abstract}

Key words COVID-19, Health system infrastructure, Brazil
Resumo Uma das preocupações ligadas à pandemia da COVID-19 se refere à capacidade da estrutura do sistema de saúde suportar a demanda por atendimento e tratamento de pessoas acometidas por esta doença. Diante disso, o objetivo deste estudo consiste em criar e mapear o Indice de Infraestrutura de Saúde (IIS) das Unidades da Federação (UFs) brasileiras, bem como verificar a sua distribuição espacial. Para isso, foi aplicada a metodologia de Análise Fatorial por Componentes Principais. Aplicou-se os testes de Bartlett e Kaiser-Meyer-Olkin para verificação da sua adequabilidade. Em seguida procedeu-se a Análise Exploratória de Dados Espaciais. Os dados foram coletados no DATASUS, COFEN, Ministério da Saúde, Portal de Compras do Governo e Portal da Transparência. Quanto aos resultados, o indice revelou que nove estados do Norte e Nordeste registraram os menores indices e cinco estados do Sudeste e Sul apresentaram os maiores indices. Foi registrado um cluster baixo-baixo nos estados do Amazonas e Pará e um Cluster alto-alto em Minas Gerais, Rio de Janeiro, São Paulo e Paraná.

Palavras-chave COVID-19, Infraestrutura do sistema de saúde, Brasil 


\section{Introdução}

Os coronavírus são um grupo de vírus de fácil contaminação e comuns aos humanos, outros mamíferos e aves, causando doenças respiratórias, hepáticas, entéricas e neurológicas. Seis espécies de coronavírus são conhecidas por causarem doenças em humanos. Dentre estes, quatro - 229E, OC43, NL63 e HKU1 - são prevalentes e normalmente causam sintomas de resfriado. Os outros dois tipos são de origem zoonótica, associados a doenças muitas vezes fatais. Trata-se do coronavírus da síndrome respiratória aguda grave (SARS-CoV) e o respiratório do Oriente Médio - coronavírus da síndrome (MERS-CoV) ${ }^{1}$. A SARS apresenta sintomas de origem infecciosa e de rápida progressão².

Em dezembro de 2019, várias unidades de saúde chinesas relataram a presença de muitos pacientes com pneumonia de causa desconhecida. Este surto, estaria associado a exposições em um mercado de frutos do mar em Wuhan, Província de Hubei, China. Em 31 de dezembro de 2019, o Centro Chinês de Controle e Prevenção de Doenças enviou uma equipe para realizar o acompanhamento e investigar, juntamente com as autoridades de saúde da província de Hubei e da cidade de Wuhan, a origem epidemiológica e etiológica dessa doença. Após este período de análise e investigação, descreveu-se a existência de um novo coronavírus em um estágio inicial de surto, os principais sintomas apresentados pelos enfermos foram febre e tosse com desconforto respiratório ${ }^{1,3}$.

Em 30 de janeiro de 2020, a OMS decretou Emergência na Saúde Pública de Interesse Internacional, decorrente do rápido aumento do número de casos e óbitos na China, provocados pela doença do novo coronavírus de 2019 (COVID-19). A partir de então, várias medidas sanitárias foram sugeridas pela OMS, tais como o isolamento horizontal das regiões afetadas, controle de temperatura em aeroportos e linhas de metrô, uso de câmeras de vigilância que ajudassem a mapear o caminho da contaminação. Ainda assim, a disseminação do vírus prosseguiu e então no dia 11 de março de 2020 decretou-se a pandemia mundial ${ }^{4}$.

Na data de 03 de julho de 2020, foram confirmados no mundo 10.710 .005 casos da COVID-19 e 517.877 mortes. Quarenta dias depois, na data de 12 de agosto de 2020, foram confirmados 20.624.316 casos de COVID-19 e 749.421 mortes, um crescimento de cerca de $48 \%$ dos casos de contaminação e $31 \%$ do número de mortes. No Brasil, o primeiro caso registrado foi em 26 de fevereiro de 2020 em São Paulo, e o primeiro óbito foi em 17 de março de 2020, neste mesmo estado $^{4}$. Na data de 03 de julho, o Brasil apresentava 1.539 .081 casos e 63.174 mortes acumulados. Até a data de 12 de agosto de 2020, foram confirmados 3.180.758 casos de COVID-19 e 104.528 mortes, um crescimento de cerca de 52\% dos casos de contaminação e $40 \%$ do número de mortes $^{5,6}$, tornando o Brasil o segundo país com o maior número de casos e mortes confirmadas por COVID-19.

Diversos países, incluindo o Brasil, têm se mobilizado para melhorar o seu sistema de saúde visando a máxima capacidade de atendimento dos pacientes que apresentarem complicações respiratórias decorrentes da contaminação pela COVID-19. De acordo com dados do DATASUS ${ }^{7}$, o Brasil possui 6.237 hospitais, destes 5.298 são hospitais de atendimento geral e 939 são especializados. Com relação aos leitos hospitalares, o Brasil possui 446.503 leitos hospitalares, destes 314.725 são leitos do Sistema Único de Saúde (SUS) (70,49\%), enquanto que 131.778 , (29,51\%) são leitos do sistema privado. A Região Sudeste concentra cerca de $40,57 \%$, seguido da Região Nordeste (27,12\%), Região Sul (16,54\%), Região Centro-oeste $(8,5 \%)$ e Região Norte $(7,27 \%)$. No entanto, Silva ${ }^{8}$ afirma que a situação hospitalar brasileira está muito longe de garantir amplo atendimento de qualidade à população, pois existem várias deficiências em sua estrutura, tais como a quantidade de leitos disponíveis, falta de medicamentos, falta de médicos, estrutura física, boa remuneração, vínculos trabalhistas legais e outros.

Conhecer o contingente de recursos e a capacidade de atendimento à saúde disponível à população de qualquer país ou região é de suma importância para o combate da COVID-19, auxiliando com a manutenção da saúde e da vida da população. Este trabalho pode ser importante ferramenta de decisão aos propositores de políticas públicas (policymakers), no que se refere a destinação de recurso público para o controle da disseminação e tratamento de pessoas contaminadas pelo vírus.

Sendo assim, o objetivo deste estudo consiste em criar e mapear o Índice de Infraestrutura de Saúde (IIS) das Unidade da Federação (UFs) brasileiras para enfrentamento da COVID-19, verificando a sua distribuição espacial. 


\section{Metodologia}

A seção está subdividida em três partes, de modo que na primeira subseção estão apresentados os dados utilizados e suas respectivas fontes. Na segunda, há uma breve apresentação do modelo multivariado, seguida dos tratamentos espaciais dos dados.

\section{Dados utilizados}

Com a finalidade de construção de um índice que permita a verificação da estrutura de enfrentamento à COVID-19 nas UFs brasileiras, foram utilizadas variáveis capazes de captar informações de diferentes dimensões em nível estadual, a saber: a estrutura física de saúde em cada UF, bem como o número de trabalhadores deste setor, a existência de dispositivos estratégicos para COVID-19 e, ainda, os recursos financeiros que foram destinados à saúde nas diferentes localidades.

As variáveis utilizadas foram coletadas do DATASUS ${ }^{9}$, do Conselho Federal de Enfermagem $(\text { COFEN })^{10}$, da Secretaria de Vigilância à Saúde (SVS), do Portal de Compras do Governo ${ }^{11}$, Ministério da Saúde ${ }^{12}$ e do Portal da transparência $^{13,14}$. No Quadro 1 estão dispostas as dimensões abordadas no estudo, as variáveis manuseadas na pesquisa e suas respectivas fontes.

Após a seleção dos dados, eles foram padronizados, o que segundo Fávero e Belfiore ${ }^{15}$ consiste em subtrair pela média e dividir pelo desvio-padrão da variável em estudo.

Nas subseções a seguir são destacados o método estatístico multivariado de análise fatorial por componentes principais para compor o índice. Em seguida, foram adotadas técnicas espaciais para o estudo da distribuição espacial do IIS. Nas subseções a seguir são destacados os procedimentos econométricos utilizados.

\section{O modelo estatístico multivariado}

Conforme mencionado, a construção do IIS se deu através da análise fatorial (AF) por componentes principais. Seguindo Fávero e Belfio$\mathrm{re}^{15}$, esta técnica permite a geração de fatores não correlacionados através de combinações lineares das variáveis iniciais. Conforme Manly ${ }^{16}$, a AF torna possível a redução de muitas variáveis em um menor número de fatores, conforme pode ser observado a seguir:

$$
X_{p}=l_{p 1} F_{1}+l_{p 2} F_{2}+\ldots+l_{p j} F_{j}+\varepsilon_{p}
$$

Onde $X_{p}$ indica a $p$-ésima variável do modelo; $l_{p}$ representa o produto da raiz quadrada dos autovalores da matriz de correlações pelos autovetores da raiz de correlações; $F_{j}$ indica os $j$-ésimos fatores e $\varepsilon_{p}$ indica a $p$-ésima combinação linear dos componentes principais $Z_{j+1}$ a $Z_{p}$.

Desta forma, as 21 variáveis utilizadas no estudo foram submetidas aos procedimentos generalizados da AF e, a partir da geração dos scores fatoriais, foi possível construir o índice de interesse. Formalmente, partindo de Mingoti ${ }^{17}$, os procedimentos adotados podem ser representados do seguinte modo:

$$
I I S_{m}=\sum_{\mathrm{j}=1}^{p}\left(\frac{\sigma_{j}^{2}}{\sum_{\mathrm{j}=1}^{p} \sigma_{j}^{2}} \mathrm{~F}_{j \mathrm{~m}}\right)
$$

Em que IIS ${ }_{m}$ é índice de infraestrutura de saúde da m-ésima Unidade de Federação (UF) brasileira, $\sigma^{2}$ é a variância explicada pelo fator $j$; $p$ é o número de fatores selecionados; $\sum_{j=1}^{p} \sigma_{j}^{2}$ representa o somatório das variâncias explicadas pelos $p$ fatores extraídos e $\mathrm{F}_{\mathrm{im}}$ é o escore fatorial da UF $m$, do fator $j$.

Antes de prosseguir, foi preciso validar a adequabilidade da metodologia de análise fatorial no estudo. Para isso, foram realizados os testes estatísticos de Kaiser-Meyer-Olkin (KMO) e o Teste de Esfericidade de Bartlett. Matematicamente, segundo Fávero e Belfiore ${ }^{15}$ os testes são representados conforme as próximas duas equações a seguir:

$$
K M O=\frac{\sum_{l=1}^{k} \sum_{c=1}^{k} \rho_{l c}^{2}}{\sum_{l=1}^{k} \sum_{c=1}^{k} \rho_{l c+}^{2} \sum_{l=1}^{k} \sum_{c=1}^{k} \varphi_{l c}^{2}}
$$

Onde $l$ são as linhas e $c$ as colunas da matriz de correlações $\rho$ das variáveis do modelo, já $\varphi$ denota os coeficientes de correlação de ordem superior das variáveis do modelo. Para a aprovação do método no teste $\mathrm{KMO}$, de acordo com literatura especializada, é recomendado que o valor seja superior a 0,6 . No entanto, o KMO pode variar entre 0 e 1 , de modo que quanto mais próximo de 1, mais adequado é o modelo.

O teste de Bartlett, por sua vez, é dado, de acordo com Fávero e Belfiore ${ }^{15}$, como:

$$
X_{\text {Bartlett }}^{2}=\left[(\mathrm{n}-1)-\left(\frac{2 k=5}{6}\right)\right] \ln |D|
$$

Onde $\mathrm{n}$ representa o tamanho da amostra, $\mathrm{k}$ o número de variáveis, $|D|$ representa o módulo do determinante da matriz de correlações $\rho$ das variáveis que compõem o índice. Para a aprova- 
Quadro 1. Informações sobre variáveis e fonte de dados da pesquisa para o ano de 2020.

\begin{tabular}{|c|c|c|c|c|}
\hline Dimensão & Variável & $\begin{array}{l}\text { Data de } \\
\text { acesso } \\
(2020)\end{array}$ & Descrição dos dados & Fonte \\
\hline \multirow[t]{6}{*}{$\begin{array}{l}\text { Estruturas } \\
\text { físicas }\end{array}$} & $\mathrm{X}_{1}$ & $11 / 06$ & $\begin{array}{l}\text { Quantidade de estabelecimentos ambulatoriais de alta } \\
\text { complexidade }^{*}\end{array}$ & \multirow[t]{6}{*}{ DATASUS $^{7}$} \\
\hline & $\mathrm{X}_{2}$ & $11 / 06$ & $\begin{array}{l}\text { Quantidade de estabelecimentos ambulatoriais de média } \\
\text { complexidade }^{\star}\end{array}$ & \\
\hline & $\mathrm{X}_{3}$ & $11 / 06$ & Quantidade de estabelecimentos ambulatoriais básicos ${ }^{*}$ & \\
\hline & $\mathrm{X}_{4}$ & $11 / 06$ & Quantidade de hospitais de alta complexidade ${ }^{*}$ & \\
\hline & $\mathrm{X}_{5}$ & $11 / 06$ & $\begin{array}{l}\text { Quantidade de estabelecimentos hospitais de média } \\
\text { complexidade }\end{array}$ & \\
\hline & $\mathrm{X}_{6}$ & $11 / 06$ & Número de postos de saúde ${ }^{\star}$ & \\
\hline \multirow{4}{*}{$\begin{array}{l}\text { Trabalhadores } \\
\text { da saúde }\end{array}$} & $\mathrm{X}_{7}$ & $12 / 06$ & Número de auxiliares de enfermagem & \multirow[t]{2}{*}{ COFEN $^{10}$} \\
\hline & $\mathrm{X}_{8}$ & $12 / 06$ & Número de enfermeiros & \\
\hline & $\mathrm{X}_{9}$ & $11 / 06$ & Número de médicos ${ }^{\star}$ & DATASUS $^{7}$ \\
\hline & $\mathrm{X}_{10}$ & $12 / 06$ & Número de técnicos em enfermagem & COFEN $^{10}$ \\
\hline \multirow{7}{*}{$\begin{array}{l}\text { Dispositivos } \\
\text { estratégicos } \\
\text { para } \\
\text { Covid-19 }\end{array}$} & $\mathrm{X}_{11}$ & 07/06 & Quantidade de testes PCR & \multirow{2}{*}{$\begin{array}{l}\text { Ministério da } \\
\text { Saúde }^{12}\end{array}$} \\
\hline & $\mathrm{X}_{12}$ & 07/06 & Quantidade de testes rápidos & \\
\hline & $\mathrm{X}_{13}$ & $11 / 06$ & Quantidades de leitos de internação não SUS* & \multirow[t]{3}{*}{ DATASUS $^{7}$} \\
\hline & $\mathrm{X}_{14}$ & $11 / 06$ & Quantidades de leitos de internação SUS* & \\
\hline & $\mathrm{X}_{15}$ & $11 / 06$ & Quantidade de respiradores e/ou ventiladores* & \\
\hline & $\mathrm{X}_{16}$ & 07/06 & Número de UTIs adulto não SUS & \multirow{2}{*}{$\begin{array}{l}\text { Ministério da } \\
\text { Saúde }\end{array}$} \\
\hline & $\mathrm{X}_{17}$ & 07/06 & Número de UTIs adulto SUS & \\
\hline \multirow[t]{4}{*}{$\begin{array}{l}\text { Recursos } \\
\text { financeiros }\end{array}$} & $\mathrm{X}_{18}$ & $11 / 06$ & $\begin{array}{l}\text { Compras nas modalidades pregão, dispensa e } \\
\text { inexigibilidade de licitação para enfrentamento da } \\
\text { Covid-19 }(\mathrm{R} \$)\end{array}$ & $\begin{array}{l}\text { Portal de } \\
\text { Compras }^{11}\end{array}$ \\
\hline & $\mathrm{X}_{19}$ & $10 / 06$ & Distribuição das despesas com saúde (R\$) & $\begin{array}{l}\text { Portal da } \\
\text { transparência }{ }^{13}\end{array}$ \\
\hline & $\mathrm{X}_{20}$ & $10 / 06$ & Valor transferido diretamente à UF $(\mathrm{R} \$)$ & \multirow{2}{*}{$\begin{array}{l}\text { Portal da } \\
\text { transparência }{ }^{14}\end{array}$} \\
\hline & $\mathrm{X}_{21}$ & $10 / 06$ & Valor transferido diretamente aos municípios $(\mathrm{R} \$)$ & \\
\hline
\end{tabular}

${ }^{*}$ Dados obtidos no Cadastro Nacional de Estabelecimentos de Saúde (CNES) foram referentes a abril de 2020, disponíveis no DATASUS.

Fonte: Elaboração própria.

ção da AF segundo esse teste, é preciso rejeitar a hipótese nula de que a matriz de correlação é uma matriz identidade.

Como frequentemente os índices são apresentados com valores dentro de um intervalo que varia de 0 a 1 , após a confirmação da adequabilidade do método e da geração dos fatores, os resultados do índice podem ser padronizados, conforme é demonstrado através da seguinte formalização:

$$
I I S_{m}=\frac{I I S_{m}-I I S_{\min }}{I I S_{\text {máx }}-I I S_{\text {min }}}
$$

Em que $I I S_{\text {min }}$ e IIS ${ }_{\text {máx }}$ são os índices mínimo e máximo calculados, respectivamente. Desta for- ma, após a padronização, o valor máximo possível do índice passa a ser 1, e o menor, 0. Logo, quanto mais próximo de 1 , maior a infraestrutura da saúde para o enfrentamento à COVID-19 da UF em questão. Para a geração dos fatores, foi utilizada a rotação fatorial ortogonal varimax que, de acordo com Hair Jr. et al. ${ }^{18}$, torna possível uma visualização mais clara de como as variáveis estão associadas e o quanto esta característica reflete de forma positiva ou negativa em cada fator.

Após a rotação ortogonal, aprovação nos testes estatísticos e a construção do índice, é possível observar a distribuição espacial do fenômeno em questão, bem como a verificação da existência de possíveis clusters, através da AEDE. Assim, seguese para o tratamento espacial dos dados. 


\section{Procedimentos de análise espacial}

Com a finalidade de verificar como se dá a distribuição no espaço do índice de Infraestrutura de saúde entre as UFs brasileiras, foram utilizadas as estatísticas I de Moran global e local. Consoante Almeida ${ }^{19}$, o I de Moran global indica se há ou não similaridade entre as variáveis em análise e o espaço. Caso o resultado dessa estatística seja positivo, então essa similaridade existe de modo que localidades com elevada incidência de uma determinada variável e/ou fenômeno, são também circuncidados por locais com alta incidência. No caso de um resultado negativo, essa relação se comporta de forma inversamente proporcional. Ou seja, localidades com altos valores são rodeados por locais com baixos valores. Já o I de Moran Local é utilizado para verificar a existência de clusters espaciais de um dado fenômeno.

O I de Moran Global na sua fórmula matricial, segundo Almeida ${ }^{19}$ é dado por:

$$
\mathrm{I}=\frac{n}{S_{o}} \frac{z^{\prime} W z}{z^{\prime} z}
$$

De modo que $n$ representa o número de regiões que são indexadas por $i \mathrm{e} j$; z representa as variáveis padronizadas; $\mathrm{W}$ representa a matriz de ponderação espacial; $\mathrm{S}_{\mathrm{o}}$ é igual a $\Sigma_{\mathrm{i}} \Sigma_{\mathrm{j}} \mathrm{w}_{\mathrm{ij}}$, indicando que as entradas da matriz de pesos espaciais $w$ serão somados; $W z$ denota a média das variáveis padronizadas nos vizinhos. Para dar prosseguimento à análise espacial, é preciso que o resultado dessa estatística aponte para a rejeição da hipótese nula de aleatoriedade espacial.

A estatística I de Moran Local foi utilizada para verificar a existência de formação de clusters espaciais do IEs $s_{\mathrm{m}}$ nas diferentes UFs do Brasil, e é dada por Anselin ${ }^{20}$ da seguinte forma:

$$
I=\sum_{i} I_{i} /\left[\mathrm{S}_{\mathrm{o}}\left(\sum_{i} z_{i}^{2} / n\right)\right]
$$

Caso o índice esteja distribuído espacialmente de modo a formar clusters, então o resultado dessa estatística aponta para a rejeição da hipótese nula de não existência de cluster espacial. Para Almeida ${ }^{19} \mathrm{o}$ Índice de Moran Local (LISA), tem como condição que a soma dos indicadores locais equivale ao global, com a devida proporção.

\section{Resultados}

A adequada aplicabilidade da análise fatorial por componentes principais foi confirmada pelos resultados dos testes KMO $(0,7459)$ e de Bartlett $(1.715,09)$. Desta forma, foi possível prosseguir para a estimativa dos fatores comuns. Através do método de rotação varimax, que maximiza a variância do fator, foi possível obter os fatores que compuseram o IIS $S_{m}$. Ao todo, foram extraídos três fatores e o critério utilizado para essa seleção foi a presença de autovalores maiores do que 1 , conforme pode ser observado na Tabela 1 .

Os três fatores extraídos, juntos, explicam cerca de $95,42 \%$ da variância total dos dados. A rotação ortogonal permitiu que fossem encontrados os coeficientes de correlação entre as 21 variáveis utilizadas e os fatores de cada uma delas. Sendo assim, os resultados das cargas fatoriais e os fatores gerados estão dispostos na Tabela 1 . Segundo literatura especializada ${ }^{17}$, é recomendada a seleção das cargas fatoriais com valor superior a, no mínimo, 0,5 .

$\mathrm{O}$ primeiro fator se mostrou relacionado com as variáveis quantidade de estabelecimentos ambulatoriais alta complexidade (x1), estabelecimentos ambulatoriais de média complexidade (x2), estabelecimentos ambulatoriais básicos (x3), hospitais de alta complexidade (x4), hospitais de média complexidade (x5), auxiliares de enfermagem (x7), enfermeiros (x8), médicos (x9), técnicos em enfermagem (x10), quantidade de testes PCR (x11), quantidade de testes rápidos (x12), quantidade de leitos de internação não SUS (x13), leitos de internação SUS (x14), respiradores e/ou ventiladores (x15), UTIs adulto não SUS (x16), UTIs adulto SUS (x17), distribuição das despesas com saúde (x19) e valor transferido aos municípios (x21). Portanto, o Fator 1 representa as estruturas gerais de funcionamento em termos de recursos físicos, humanos e de material.

O segundo fator, por sua vez, se relacionou com as variáveis, hospitais de média complexidade (x5), número de postos de saúde (x6) e valor transferido diretamente à UF (x20). Este fator representa mecanismos iniciais de acesso à atendimento médico e fonte de recursos diretos estaduais. Por fim, o Fator 3 está relacionado com as variáveis quantidades de testes PCR (x11), compras nas modalidades por pregão, dispensa e inexigibilidade de licitação para enfrentamento da COVID-19 (x18) e valor transferido diretamente à UF (x20). Assim, o terceiro fator formou-se no contexto da pandemia da COVID-19, como con- 
Tabela 1. Cargas fatoriais, comunalidades, variáveis, autovalores, variâncias por fator.

\begin{tabular}{|c|c|c|c|c|}
\hline Variável & Fator 1 & Fator 2 & Fator 3 & Comunalidade \\
\hline $\mathrm{X} 1$ & $0,9427^{\star}$ & 0,2257 & 0,1700 & 0,9685 \\
\hline $\mathrm{X} 2$ & $0,9563^{\star}$ & 0,2314 & 0,0045 & 0,9681 \\
\hline $\mathrm{X} 3$ & $0,9207^{\star}$ & 0,3200 & $-0,0147$ & 0,9503 \\
\hline $\mathrm{X} 4$ & $0,9180^{*}$ & 0,2249 & 0,2988 & 0,9826 \\
\hline $\mathrm{X} 5$ & $0,8197^{\star}$ & $0,5201^{\star}$ & 0,0015 & 0,9425 \\
\hline X6 & 0,2070 & $0,9351^{\star}$ & $-0,0741$ & 0,9227 \\
\hline $\mathrm{X} 7$ & $0,9494^{\star}$ & $-0,0736$ & 0,1284 & 0,9232 \\
\hline X8 & $0,9546^{\star}$ & 0,1691 & 0,2088 & 0,9835 \\
\hline X9 & $0,9783^{\star}$ & 0,1139 & 0,1609 & 0,9960 \\
\hline $\mathrm{X} 10$ & $0,8392^{*}$ & 0,3194 & 0,4158 & 0,9791 \\
\hline $\mathrm{X} 11$ & $0,6132^{\star}$ & 0,2269 & $0,6987^{\star}$ & 0,9158 \\
\hline $\mathrm{X} 12$ & $0,9501^{*}$ & 0,2676 & 0,1341 & 0,9923 \\
\hline $\mathrm{X} 13$ & $0,9831^{\star}$ & 0,1139 & 0,1187 & 0,9935 \\
\hline X14 & $0,9113^{\star}$ & 0,3798 & 0,0961 & 0,9839 \\
\hline X15 & $0,9583^{\star}$ & 0,1125 & 0,2551 & 0,9960 \\
\hline $\mathrm{X} 16$ & $0,9085^{\star}$ & 0,0341 & 0,3953 & 0,9827 \\
\hline $\mathrm{X} 17$ & $0,9713^{\star}$ & 0,1875 & 0,0403 & 0,9802 \\
\hline $\mathrm{X} 18$ & 0,0280 & $-0,1663$ & $0,8877^{\star}$ & 0,8164 \\
\hline X19 & $0,8659^{*}$ & 0,2166 & 0,3522 & 0,9207 \\
\hline $\mathrm{X} 20$ & 0,1287 & $0,6369^{\star}$ & $0,6591^{\star}$ & 0,8567 \\
\hline $\mathrm{X} 21$ & $0,8634^{*}$ & 0,4595 & 0,1624 & 0,9829 \\
\hline Autovalores & 14,9575 & 2,5721 & 2,5081 & - \\
\hline Variância explicada (\%) & 0,7123 & 0,1225 & 0,1194 & - \\
\hline Variância acumulada (\%) & 0,7123 & 0,8347 & 0,9542 & - \\
\hline
\end{tabular}

${ }^{*}$ Cargas fatoriais maiores que 0,5 foram marcadas por um asterisco. Fonte: Elaboração própria.

tribuinte para a explicação do funcionamento hospitalar nas UFs brasileiras, diante elevada demanda populacional por atendimento de saúde.

Após a formação dos fatores, seguiu-se para a padronização dos resultados para a formação do índice. Assim, foi construído um índice para cada UF do país. O maior Índice de Infraestrutura de Saúde foi encontrado no estado de São Paulo (0,781795), seguido pelo estado de Minas Gerais (0,352699), ambos na região Sudeste. Os menores índices, por sua vez, foram registrados no Amapá $(0,045114)$ e em Roraima $(0,045722)$, na região Norte do país. Na Tabela 2 é possível observar o IIS $S_{m}$ de cada UF do Brasil.

Os resultados numéricos dos índices sugerem que a distribuição espacial da estrutura do sistema de saúde no Brasil não se dá de forma homogênea. Em exemplo, apenas o estado de São Paulo alcançou um índice superior a 0,5. Ademais, é possível observar disparidades regionais no que tange à essa distribuição. Enquanto os maiores índices concentram-se na região Sudeste, os me- nores estão presentes na região Norte, onde houve relatos de sobrecarga nos sistemas de saúde e funerário, ocasionados pela COVID-1921.

Em prol de uma melhor observação do agrupamento dos $I I S_{m}$ nas Unidades da Federação, na Figura 1, encontra-se a disposição espacial dos índices nas diferentes localidades do país. Estes resultados foram obtidos através do método hierarchical clustering, com indicação de formação de seis agrupamentos. Construídos mediante o método Ward linkage, com aplicação de distância euclidiana. Para a obtenção desses grupos, partiu-se dos resultados dos índices, conforme apresentado na Tabela 2 .

A Figura 1 organiza os grupos de modo que, quanto maior o índice, mais escura é a tonalidade representada no mapa. Desta forma, é possível observar que os estados do Amapá (0,045114), Roraima (0,045722), Acre (0,045823), Rondônia $(0,06500)$, Sergipe $(0,067870)$ e Tocantins $(0,057963)$ compõem o grupo dos menores índices, ou seja, o Grupo 1. 
Tabela 2. Resultados dos Índice de Infraestrutura de Saúde $\left(I I S_{m}\right)$ por UF e região, em 2020.

\begin{tabular}{llllll}
\hline \multicolumn{1}{c}{ Região } & \multicolumn{1}{c}{ UF } & \multicolumn{1}{c}{$\boldsymbol{I I S}_{\boldsymbol{m}}$} & \multicolumn{1}{c}{ Região } & \multicolumn{1}{c}{ UF } & $\boldsymbol{I I S}_{\boldsymbol{m}}$ \\
\hline Norte & Rondônia & 0,065007 & Nordeste & Sergipe & 0,067870 \\
Norte & Acre & 0,045823 & Nordeste & Bahia & 0,218160 \\
Norte & Amazonas & 0,080364 & Sudeste & Minas Gerais & 0,352699 \\
Norte & Roraima & 0,045722 & Sudeste & Espírito Santo & 0,096621 \\
Norte & Pará & 0,118611 & Sudeste & Rio de Janeiro & 0,332393 \\
Norte & Amapá & 0,045114 & Sudeste & São Paulo & 0,781795 \\
Norte & Tocantins & 0,057963 & Sul & Paraná & 0,233039 \\
Nordeste & Maranhão & 0,109346 & Sul & Santa Catarina & 0,156648 \\
Nordeste & Piauí & 0,076482 & Sul & Rio Grande do Sul & 0,229693 \\
Nordeste & Ceará & 0,147787 & Centro-Oeste & Mato Grosso do Sul & 0,082707 \\
Nordeste & Rio Grande do Norte & 0,085771 & Centro-Oeste & Mato Grosso & 0,096832 \\
Nordeste & Paraíba & 0,094154 & Centro-Oeste & Goiás & 0,149312 \\
Nordeste & Pernambuco & 0,167269 & Centro-Oeste & Distrito Federal & 0,110340 \\
Nordeste & Alagoas & 0,076142 & & & \\
\hline
\end{tabular}

Fonte: Elaboração própria, a partir de dados da pesquisa e $\mathrm{IBGE}^{27}$.

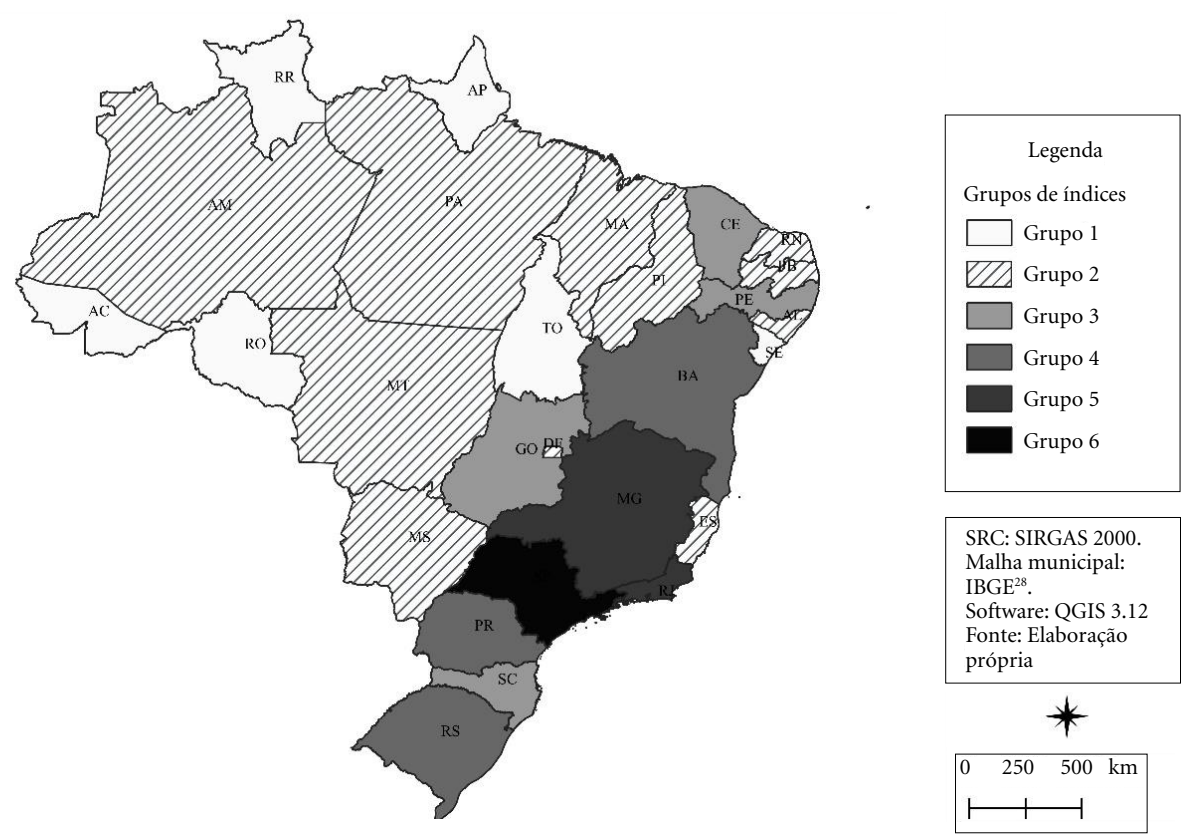

Figura 1. Disposição espacial dos índices por agrupamento.

O Grupo 2 é formado pelos estados do Amazonas (0,080364), Pará (0,118611), Maranhão (0,109346), Piauí (0,07648), Rio Grande do Norte (0,085771), Paraíba (0,094154), Alagoas (0,076142), Espírito Santo (0,09662), Mato Gros- so $(0,096832)$, Mato Grosso do Sul $(0,082707)$ e Distrito Federal $(0,11034)$.

No terceiro grupo encontram-se os estados de Pernambuco $(0,16726)$, Ceará $(0,147787)$, Goiás $(0,149312)$ e Santa Catarina $(0,156648)$. 

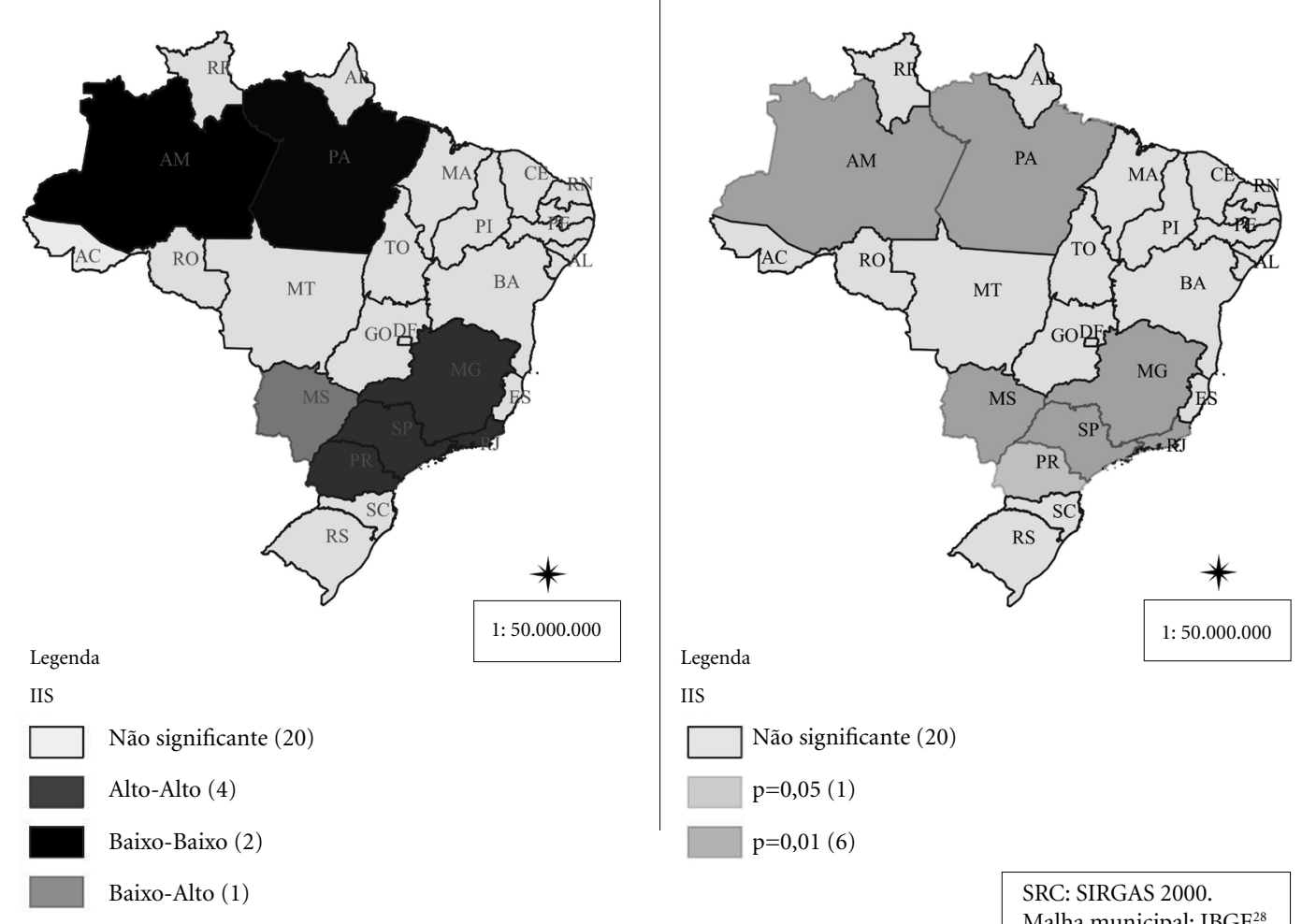

SRC: SIRGAS 2000.

Malha municipal: $\mathrm{IBGE}^{28}$

Software: QGIS 3.12

Figura 2. Clusters e nível de significância alcançados através da AEDE.

Fonte: Elaboração própria

Enquanto isso, o Grupo 4 é composto pela Bahia $(0,218160)$, pelo Paraná $(0,233039)$ e pelo Rio Grande do Sul $(0,229693)$.

Os grupos com os maiores valores do IIS encontram-se nos grupos 5 e 6 . O quinto grupo é formado pelo Rio de Janeiro $(0,33239)$ e por Minas Gerais $(0,352699)$. Destaca-se o fato de que o Grupo 6 abarca somente o estado de São Paulo (0,781795).

Apesar da distribuição espacial do índice nas diferentes UFs se comportar de forma dessemelhante, faz-se necessária a verificação da existência de padrões de dependência espacial. Para tal, utilizam-se os ferramentais estatísticos I de Moran global e I de Moran local.

Com a intenção de obtenção das evidências acerca da correlação espacial, foi calculado o I de Moran global univariado. No cálculo, foram utilizadas as convenções de matriz de contiguidade queen (rainha) - os resultados para a matriz rook (torre) foram semelhantes. Os resultados foram capazes de confirmar que o IIS ${ }_{m}$ é autocorrelacionado espacialmente entre as UFs brasileiras, na medida em que a hipótese nula de aleatoriedade espacial foi rejeitada.

Confirmada a dependência espacial, foi possível analisar os padrões locais de associação espacial da infraestrutura de saúde de enfrentamento à COVID-19 no país. Através do cálculo do I de Moran local, observou-se a formação de clusters do tipo baixo-baixo na região Norte do Brasil e do tipo alto-alto na região Sudeste. Ademais, encontrou-se um padrão de aglomeração do tipo baixo-alto no estado do Mato Grosso do Sul. Na Figura 2 é possível observar a formação dos clusters no território brasileiro. Considera-se p-valor menor ou igual a 5\% como indicador de significância estatística.

A existência de um cluster do tipo baixo-baixo significa que UFs com um baixo IIS $_{m}$ estão também circundados por outros UFs com baixo $I I S_{m}$. No caso do cluster do tipo alto-alto, o in- 
verso ocorre. Ou seja, UFs com elevada infraestrutura de saúde estão rodeados por outras UFs com características semelhantes neste aspecto, isto é, também com alta infraestrutura de saúde. O padrão de cluster tipo baixo-alto, por sua vez, indica que UFs com baixo IIS ${ }_{m}$ estão cercados por outros estados que possuem um IIS ${ }_{m}$ alto.

O padrão de clusters baixo-baixo envolve os estados do Amazonas e do Pará. Já o padrão alto-alto foi observado, sobretudo, nas regiões Sul e Sudeste. Os estados de Minas Gerais, Rio de Janeiro, São Paulo e Paraná compuseram essa aglomeração. O estado do Mato Grosso do Sul, conforme anteriormente mencionado, formou um cluster do tipo baixo-alto.

\section{Discussão}

A construção do índice buscou evidenciar a distribuição da infraestrutura de saúde no Brasil. Os valores obtidos revelam a heterogeneidade $\mathrm{e}$ a desigualdade de acesso da população brasileira ao atendimento no sistema de saúde, evidenciadas pela amplitude da primeira (São Paulo $I I S_{m}=$ 0,781795) e última (Amapá $I I S_{m}=0,045114$ ) unidade de federação em termos de posição no $I I S_{m}$. É possível notar que algumas unidades de federação se agruparam com outras de diferentes regiões, mesmo que não sejam estritamente vizinhas. Dentro de uma região há diversas realidades, com escalas diferentes de infraestrutura de saúde.

Ressalta-se que as UFs dos agrupamentos $1 \mathrm{e}$ 2 - região Norte, e parte das regiões Nordeste e Centro-Oeste - possuem baixos níveis de infraestrutura de saúde, em comparação às UFs dos demais agrupamentos. Este padrão também pode ser percebido em termos de clusters espaciais estatisticamente significativos. Desta forma é preciso atenção ao Amazonas e ao Pará, por formarem um cluster baixo-baixo em termos do IIS ${ }_{m}$, o que pode significar maior fragilidade destes locais no que se refere à capacidade de atendimento à população, principalmente em situações críticas, de choque elevado de demanda.

A situação das localidades com baixa infraestrutura de saúde pode ser agravada pela distância entre essas UFs e aquelas com maior $I I S_{m}$, para uma possível tentativa de transferência de pacientes graves para outras unidades de saúde, por exemplo. Smolski et al. ${ }^{22}$, em uma análise do Rio Grande do Sul, suas Regiões de Saúde e seus respectivos municípios, com relação à disponibilidade de leitos hospitalares no enfrentamento da
COVID-19, concluíram que a maior parte da população é dependente de deslocamentos para ter acesso a este tipo de serviço de saúde, o qual está concentrado em centros de referência regional; além disso, foi observado que há desigualdades na distribuição e oferta de leitos hospitalares e respiradores/ventiladores; e, em algumas Regiões de Saúde nem sequer foram encontrados registros de leitos de UTI do tipo Adulto. Pedrosa e Albuquerque $^{23}$ também buscaram analisar a distribuição dos casos da COVID-19 e dos leitos de unidade de terapia intensiva (UTI), destinados exclusivamente à doença, porém no estado brasileiro do Ceará.

Uma boa infraestrutura para o enfrentamento de episódios de alta demanda pelos serviços de saúde, como é o caso da pandemia da COVID-19, pode ser fundamental no combate à mortalidade pela doença. Uma evidência disso é o estudo de Moreira ${ }^{24}$, que buscou identificar regiões com maiores taxas de mortalidade específica decorrentes da COVID-19, e locais de maior escassez de leitos de UTI e ventiladores pulmonares. Os principais resultados apontaram para quatro perfis em potencial de mortalidade, formado pelo conjunto de Regiões de Saúde com coeficientes baixos; valores médios; coeficientes altos; e coeficientes muito altos. As regiões de saúde que apresentaram as maiores médias na mortalidade, localizam-se em locais cuja escassez de leitos de UTI e de ventiladores foi observada, principalmente, em partes das regiões Nordeste, Sudeste e Sul.

Para Noronha et al..$^{25}$ diante da pandemia da COVID-19 no Brasil, a pressão por atendimentos, evidencia mais a desigualdade de oferta de serviços de saúde, insuficientes, mesmo com a presença do setor privado. O reforço da estrutura deve ser feito, por exemplo, com hospitais de campanha, combinados a estratégias da redução da velocidade de contaminação.

Os maiores valores do índice de infraestrutura de saúde construído foram localizados, sobretudo, na região Sudeste e, mais especificamente, em São Paulo. Sublinha-se aqui as informações da Agência Nacional de Saúde Suplementar (ANS) ${ }^{26}$ para março de 2020, onde foi verificado que a região Sudeste é a região que conta com o maior número de beneficiários de planos de saúde, medidos por número de vínculos firmados com instituições de saúde suplementar. Em seguida, encontram-se as regiões Sul, Nordeste, Centro-Oeste e por fim, a região Norte. Ademais, segundo a Pesquisa de Orçamentos Familiares (POF) de 2008, as famílias da região Sudeste são 
as com maiores despesas médias com assistência à saúde e planos de saúde, seguidas pelas regiões Sul, Centro-Oeste, Nordeste e Norte ${ }^{27}$.

Importante destacar que o nível de infraestrutura de saúde não reflete isoladamente o nível de vulnerabilidade da UF em relação a pandemias, como a de COVID-19, uma vez que fatores como velocidade de propagação do vírus, índice de isolamento, uso de máscaras, outras medidas que reduzam o contágio, quantidade de habitantes, distância de moradias e a quantidade de pessoas por domicílio, possivelmente poderiam interferir. Estes elementos podem ser avaliados em trabalhos posteriores.

Espera-se que este estudo contribua com as políticas públicas voltadas à ampliação da infraestrutura de saúde no Brasil, em especial com a finalidade de atenuar as existentes disparidades regionais. Dentre as limitações deste estudo, des- tacam-se as poucas produções científicas acerca da pandemia da COVID-19 e de seu comportamento em nível nacional, visto que é um fenômeno recente e ainda de difícil mensuração de seus efeitos, o que limita a discussão acerca do assunto. Outras limitações associam-se a estruturas de saúde recém-criadas no período da pandemia, o que dificulta a contemplação de todas as informações no estudo. Além disso, é preciso ponderar que a infraestrutura da saúde é destinada à prevenção, diagnóstico e tratamento de diversas doenças, não apenas à COVID-19, então os resultados obtidos devem ser analisados com parcimônia, uma vez que o sistema de saúde deve ser preparado para o enfrentamento de diversos tipos de enfermidades. Recomenda-se que futuros trabalhos ampliem o estudo através de informações municipais e ao longo do tempo, com índices dinâmicos.

\section{Colaboradores}

Todos os autores participaram igualmente de todas as etapas de execução do trabalho, desde a ideia inicial, até a escrita e revisão do artigo. 


\section{Referências}

1. Zhu N, Zhang D, Wang W, Li X, Yang B, Song J, Zhao X, Huang B, Shi W, Lu R, Niu P, Zhan F, Ma X, Wang D, Xu W, Wu G, Gao GF, Tan W, China Novel Coronavirus Investigating and Research Team. A novel coronavirus from patients with pneumonia in China, 2019. N Engl J Med 2020; 382(8):727-733.

2. Tsang KW, Ho PL, Ooi GC, Yee WK, Wang T, Chan -Yeung M, Lam WK, Seto WH, Yam LY, Cheung TM, Wong PC, Lam B, Ip MS, Chan J, Yuen KY, Lai KN. A cluster of cases of severe acute respiratory syndrome in Hong Kong. N Engl J Med 2003; 348(20):1975-1983.

3. World Health Organization (WHO). Novel coronavirus: China, 2020 [Internet]. [acessado 2020 Jun 26]. Disponível em: http://www.who.int/csr/don/12-january-2020-novel-coronavirus-china/en/

4. Organização Pan-Americana da Saúde (OPAS). Folha informativa - COVID-19: doença causada pelo novo coronavírus, 2020 [Internet]. [acessado 2020 Jul 04]. Disponível em: https://www.paho.org/bra/index. php?option $=$ com_content\&view $=$ article $\&$ id $=6101$ : covid19\&Itemid $=875$

5. Brasil. Ministério da Saúde (MS). Coronavírus - COVID 19: O que você precisa saber [Internet]. 2020 [acessado 2020 Jun 26]. Disponível em: https://coronavirus.saude.gov.br/

6. Brasil. Ministério da Saúde (MS). COVID-19 no Brasil [Internet]. 2020 [acessado 2020 Jul 4]. Disponível em: http://susanalitico.saude.gov.br/\#/dashboard/

7. Brasil. Ministério da Saúde (MS). DATASUS. Tabnet [Internet]. [acessado 2020 Jun 11]. Disponível em: https://datasus.saude.gov.br/informacoes-de-saudetabnet/

8. Silva HB. Mais Médicos e o Médicos pelo Brasil, 2019 [Internet]. [acessado 2020 Jun 26]. Disponível em: http://www.portal.cfm.org.br/index.php? option $=$ com_content $\&$ view $=$ article $\&$ id $=28381$ : 2019-08-09-19-00-50\&catid=46:artigos\&Itemid $=18$

9. Brasil. Ministério da Saúde (MS). DATASUS. Leitos de internação [Internet]. [acessado 2020 Jun 26]. Disponível em: http://tabnet.datasus.gov.br/cgi/deftohtm. exe?cnes/cnv/leiintbr.def

10. Conselho Federal de Enfermagem (COFEN). Enfermagem em números [Internet]. [acessado 2020 Jun 12]. Disponível em: http://www.cofen.gov.br/enfermagem-em-numeros

11. Portal de Compras. Painel de compras COVID-19 [Internet]. 2020 [acessado 2020 Jun 11]. Disponível em: https://www.comprasgovernamentais.gov.br/index. $\mathrm{php} /$ transparencia/60-transparencia/1313-transparencia-dos-dados-de-compras-para-o-covid-19

12. Barsil. Ministério da Saúde (MS). Painel de leitos e insumos [Internet]. 2020 [acessado 2020 Jun 7]. Disponível em: https://covidinsumos.saude.gov.br/paineis/ insumos/painel.php

13. Portal da Transparência. Saúde: Distribuição das despesas com saúde por localidade [Internet]. 2020 [acessado 2020 Jun 10]. Disponível em: http://www.portaltransparencia.gov.br/funcoes/10-saude? $a n o=2020$

14. Portal da Transparência. Recursos transferidos [Internet]. 2020 [acessado 2020 Jun 10]. Disponível em: http://www.portaldatransparencia.gov.br/transferencias.
15. Fávero LP, Belfiore P. Manual de análise de dados. Rio de Janeiro: Elsevier; 2017.

16. Manly BFJ. Métodos estatísticos multivariados: uma introdução. $3^{\mathrm{a}}$ ed. Porto Alegre: Bookman; 2008.

17. Mingoti SA. Análise de dados através de métodos de estatística multivariada: uma abordagem aplicada. Belo Horizonte: Editora UFMG; 2005.

18. Hair Jr JF, Black WC, Babin BJ, Anderson RE, Tatham RL. Análise multivariada de dados. Porto Alegre: Bookman Editora; 2009.

19. Almeida E. Econometria espacial. Campinas: Alínea; 2012.

20. Anselin L. Local Indicators of Spatial Association LISA. Geographical Analysis 1995; 27(2):93-115.

21. Silva SG. Pandemia e afetações das emoções: reflexões sobre a realidade da Covid-19 no estado do Amapá. Rev Bras Sociol Emoção 2020; 19(55):113-124.

22. Smolski FMS, Battisti IDE, Soder RM, Rotta E, Kucharski KW. Disponibilidade de leitos hospitalares e ventilação mecânica no Rio Grande do Sul: desafios no enfrentamento da COVID-19 Internet]. 2020 [acessado 2020 Jun 25]. Disponível em: https://www. researchgate.net/publication/341651488_Disponibilidade_de_leitos_hospitalares_e_ventilacao_mecanica_no_Rio_Grande_do_Sul_desafios_no_enfrentamento_da_COVID-19

23. Pedrosa NL, Albuquerque NLS. Análise Espacial dos Casos de COVID-19 e leitos de terapia intensiva no estado do Ceará, Brasil. Cien Saude Colet 2020; 25(Supl. 1):2461-2468.

24. Moreira RS. COVID-19: unidades de terapia intensiva, ventiladores mecânicos e perfis latentes de mortalidade associados à letalidade no Brasil. Cad Saude Publica 2020; 36(5):e00080020.

25. Noronha KVMS, Guedes GR, Turra CM, Andrade MV, Botega L, Nogueira D, Calazans JÁ, Carvalho L, Servo L, Ferreira MF. Pandemia por COVID-19 no Brasil: análise da demanda e da oferta de leitos hospitalares e equipamentos de ventilação assistida segundo diferentes cenários. Cad Saude Publica 2020; 36(6):e00115320.

26. Agência Nacional de Saúde Suplementar (ANS). Beneficiários [Internet]. [acessado 2020 Jun 29]. Disponível em: http://www.ans.gov.br/anstabnet/cgi-bin/ dh?dados/tabnet_br.def

27. Instituto Brasileiro de Geografia e Estatística (IBGE). Sistema IBGE de Recuperação Automática [Internet]. [acessado 2020 Jun 28]. Disponível em: https://sidra. ibge.gov.br

28. Instituto Brasileiro de Geografia e Estatística (IBGE). Malhas municipais 2019 [Internet]. [acessado 2020 Jun 6]. Disponível em: https://www.ibge.gov.br/geociencias/organizacao-do-territorio/15774-malhas. html? $=\& \mathrm{t}=$ downloads.

Artigo apresentado em 16/07/2020

Aprovado em 13/09/2020

Versão final apresentada em 15/09/2020 
